\title{
Exchange Explosions: Magnetization Dynamics during Vortex-Antivortex Annihilation
}

\author{
Riccardo Hertel and Claus M. Schneider \\ Institut für Festkörperforschung IFF-9 "Elektronische Eigenschaften," Forschungszentrum Jülich GmbH, D-52425 Jülich, Germany
}

(Received 31 July 2006; published 23 October 2006)

\begin{abstract}
A magnetic vortex and an antivortex can annihilate, resulting in a homogeneous magnetization. A detailed description of the magnetization dynamics of such annihilation processes is obtained by micromagnetic simulations based on the Landau-Lifshitz-Gilbert equation. We show that, depending on the relative polarization of the vortex-antivortex pair, the annihilation process is either a continuous transformation of the magnetic structure or it involves the propagation of a micromagnetic singularity (Bloch point) causing a burstlike emission of spin waves. These results provide new insight into a fundamental micromagnetic process that has recently been proposed for a controlled generation of spin waves.
\end{abstract}

DOI: 10.1103/PhysRevLett.97.177202

PACS numbers: $75.40 . \mathrm{Gb}, 75.40 . \mathrm{Mg}, 75.75 .+\mathrm{a}$

Magnetic vortices in ferromagnets are regions of typically just a few nanometers size, with a core around which the magnetization circulates. Up to a few years ago, such magnetic vortices were considered mainly as a topological detail of magnetic flux-closure patterns [1]. It was found that vortices inevitably occur in singly connected samples with magnetic flux-closure patterns and that they may be located, e.g., at the junctions of magnetic domains [2]. In the wake of the recent dramatic progress in nanomagnetism, static and dynamic properties of submicrometer-sized particles have been extensively studied, and the tiny magnetic vortices and particularly their dynamic properties have moved into the focus of interest [3-7]

The counterpart of a magnetic vortex is a magnetic structure with similar properties, known as an antivortex. Both vortex and antivortex have a magnetic core which is magnetized perpendicular to the plane. A vortex and an antivortex can annihilate when they meet $[8,9]$. Besides the fact that the annihilation is connected with an emission of spin waves [10], not much is known about the magnetization dynamics of a vortex-antivortex annihilation process. We have studied the annihilation of a vortex and an antivortex with finite-element micromagnetic simulations based on the Landau-Lifshitz-Gilbert equation. These simulations yield a detailed description of this previously unexplored fundamental magnetization process. The results reveal the process to strongly depend on the relative orientation of the core magnetization of both the vortex and the antivortex. Particularly, if the vortex and antivortex cores are antiparallel to each other, the annihilation process involves the propagation of a Bloch point, which causes a burstlike dissipation of exchange energy ("exchange explosion") via spin waves. Considering the proposed use of magnetic vortices in data storage and magnetologic approaches $[5,11,12]$, a good control of the dynamic behavior will be mandatory. Understanding the annihilation dynamics of vortices and antivortices is, therefore, an important step towards a precise description of the complicated dynamic magnetization processes involving the temporary formation of vortices.
The similarities and differences between a vortex and an antivortex are shown schematically in Fig. 1. Along the path of a closed loop around the core of a vortex or an antivortex, the in-plane magnetization direction rotates by $360^{\circ}$. The two structures can be distinguished by the winding number $W=\oint[\alpha(\phi) / 2 \pi] d S$, i.e., the normalized line integral on a closed loop $S$ over the angle $\alpha$ that the magnetization $\vec{M}$ encloses with the $x$ axis. The value of the winding number is +1 in the case of a vortex and -1 for an antivortex. Unless a vortex enters through the boundary of the sample, the winding number of a thin-film element is a topological invariant [13]. Hence, magnetic vortices and antivortices can only be created or annihilated pairwise and never individually. The perpendicular orientation of $\vec{M}$ in the vortex and antivortex cores is due to the ferromagnetic exchange interaction. A full in-plane magnetization would lead to a singularity of the exchange energy density, which is avoided if $\vec{M}$ turns out of the plane. The orientation of the magnetization in the core is often called the polarization. Unlike the vortex structure, which develops naturally, e.g., in soft-magnetic nanodisks [14], an isolated antivortex is not stable. Antivortices can occur in cross-tie domain wall structures $[15,16]$, where they are enclosed by two vortices with the same sense of rotation.

We have simulated the annihilation of an antivortex and a vortex in a cross-tie domain wall structure in a square Permalloy thin-film element (exchange $A=13 \mathrm{pJ} / \mathrm{m}$, saturation magnetization $M_{s}=795 \mathrm{kA} / \mathrm{m}$, Gilbert damping constant $\alpha=0.01$ ) of $100 \mathrm{~nm} \times 100 \mathrm{~nm}$ size and

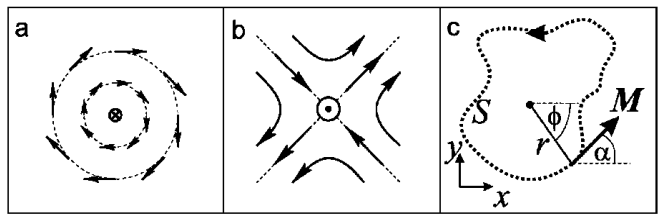

FIG. 1. Schematics of a magnetic (a) vortex and (b) an antivortex structure. In the core of the (anti)vortex, the magnetization is perpendicular to the plane. (c) Schematic representation on the definition of the winding number. 
$10 \mathrm{~nm}$ thickness. A Permalloy platelet of this size is too small to permanently sustain a cross-tie structure. If such a magnetic structure is imposed as an initial magnetic configuration, it decays into a symmetric vortex state. This domain structure conversion can occur only via a vortexantivortex annihilation process. At zero field, the conversion into a vortex state occurs within $\sim 200$ ps. The simulations were performed with the micromagnetic finiteelement code based on the Landau-Lifshitz-Gilbert equation already used in previous studies [17]. The finite element mesh consisted of about 200000 tetrahedral elements of almost equal size. The largest distance between neighboring discretization points was $1.3 \mathrm{~nm}$.

The results show that the annihilation process is relatively simple if the vortices are polarized parallel to the polarization of the antivortex. In this case, the cores of the vortices approach each other on spiralling orbits and meet in the center. During this approach, the in-plane magnetization between the antivortex and the vortex continuously rotates out of plane, thereby dissolving the complicated initial magnetization structure. Because of this continuous transition, it is difficult to identify at which point in time the vortex-antivortex annihilation occurs. The usual representation of magnetic domain structures in thin-film elements consists in displaying one magnetization component with a color code or a gray scale. This corresponds to what would be observable, e.g., in a Kerr microscopy image of a larger sample. Such a representation of $m_{y}$ is shown in Figs. 2(a)-2(e). In Figs. 2(a) and 2(b), the approximate position of the vortices and the antivortex is indicated with circles and with a cross, respectively. A much more precise localization of the vortices and the antivortex can be obtained using isosurfaces [18]: The cores of the vortex and the antivortex are the only points in the sample where the $z$ component of the magnetization reaches $100 \%$. In other words, both the $x$ and $y$ components are zero at the vortex cores. Therefore, the cores are located where the isosurfaces $m_{x}=0$ and $m_{y}=0$ intersect. In Figs. 2(f) $-2(\mathrm{j})$, the cores are encircled by cylindrical isosurfaces displaying the regions where $m_{z}=90 \%$. Using this representation with isosurfaces, the spiralling motion of the vortices around the center of the sample during the relaxation can be seen very clearly. As the vortices approach the antivortex, after about 90 ps [19] the three $m_{z}=90 \%$ isosurfaces merge into one single ribbon that surrounds the intersection of the $m_{x}=0$ and $m_{y}=0$ surfaces [Fig. 2(i)]. Both vortices and the antivortex are involved in this process. The annihilation of the antivortex is a smooth, continuous rearrangement of the magnetization [20]. As can be seen in Fig. 4, the energy of the system decreases continuously during the process. The actual annihilation process, where the antivortex meets the vortices, does not appear as a welldefined event.

The situation is very different in the case of opposite polarization of the antivortex with respect to the polarization of the neighboring vortices. The initial configuration is again a cross-tie structure as displayed in Figs. 2(a) and $2(\mathrm{f})$, but now the polarization of the antivortex is negative, while the polarization of the vortices is positive. Using the representation with isosurfaces as described before, the dynamics of this annihilation process is shown in Fig. 3. The blue cylinder around the antivortex is the isosurface of $m_{z}=-90 \%$, and the red cylinders around the vortex cores are isosurfaces of $m_{z}=+90 \%$. The highly symmetric cross-tie structure represents an unstable equilibrium as a starting point, where the antivortex can move either to the left or to the right. To prevent this ambiguity, the path of the annihilation is determined uniquely by introducing a small break of symmetry in the initial configuration: The position of the antivortex core is shifted by about $2 \mathrm{~nm}$ to the left of the center.

Similar to the previous case, the vortex cores move in the plane on curved orbits around the center. At the same time, the antivortex further approaches the vortex on the left. As the distance between the vortex and the antivortex with opposite polarization is reduced, the cores of the vortex and
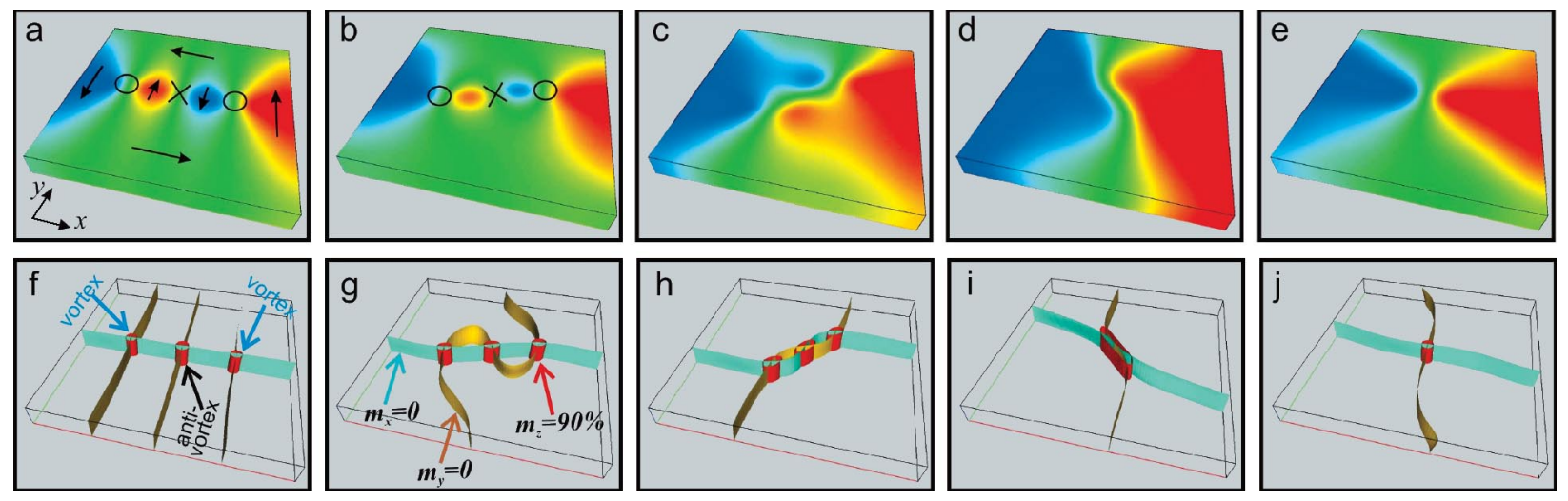

FIG. 2 (color). Magnetization dynamics of the domain structure conversion from a cross-tie wall structure to a vortex state. The polarization of the vortices is parallel to the one of the central antivortex. Ten snapshots are taken at five different times [(a),(f): 0 ps; (b),(g): $30 \mathrm{ps;} \mathrm{(c),(h):} 70 \mathrm{ps;} \mathrm{(d),(i):} 90 \mathrm{ps;} \mathrm{(e),(j):} 125 \mathrm{ps}]$. In (a)-(e), the $y$ component of the magnetization is shown with a color code going from blue $(-100 \%)$ over green $(0 \%)$ to red $(+100 \%)$. In the bottom row, the same magnetic structures are displayed with an isosurface representation. The meaning of the isosurfaces is described in more detail in the text. 

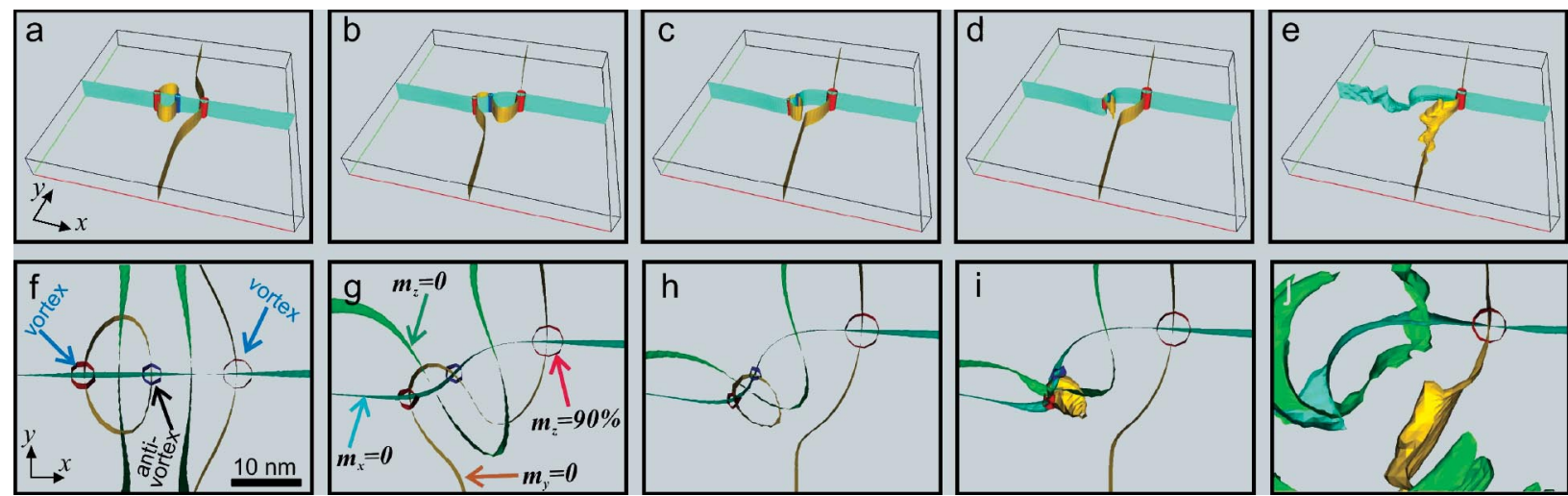

FIG. 3 (color). Dynamics of a vortex-antivortex annihilation process with opposite polarization. In (a)-(e), a visualization with isosurfaces has been used, as done previously in the bottom row in Fig. 2. The configurations are shown at (a),(f) 5 ps, (b),(g) 110 ps, (c),(h) $135 \mathrm{ps}$, (d),(i) $138 \mathrm{ps,} \mathrm{and} \mathrm{(e),(j)} 141$ ps. In the bottom row, (f)-(j) show magnified top views on the region of interest. In addition, the isosurfaces $m_{z}=0$ are displayed as green ribbons in these panels.

the antivortex become much sharper. This can be seen in Figs. 3(g) and 3(h), which show that the diameter of the cylindrical $m_{z}= \pm 90 \%$ isosurface is reduced to about $2 \mathrm{~nm}$, i.e., less than half of the size compared to the vortex on the right. Note that in Fig. 3(h) the distance between the vortex and the antivortex has reduced to about $4 \mathrm{~nm}$. The occurrence of such strongly inhomogeneous magnetic structures on length scales considerably below the exchange length $[21,22] l_{s}=\sqrt{2 A /\left(\mu_{0} M_{s}^{2}\right)}$ is quite surprising. Usually, the exchange length is considered to be the smallest relevant length scale in structurally homogeneous ferromagnetic materials. However, there are exceptional cases where large deviations of the magnetization can occur in a ferromagnet even on atomic distances [23]. The approach of the vortex and the antivortex proceeds until their cores meet in one point, leading to a dramatic and sudden annihilation process. The annihilation is accomplished by a micromagnetic singularity [23] (Bloch point) that propagates vertically through the sample. The propagating Bloch point (BP) dissolves simultaneously both the vortex and the antivortex structure. In the continuum theory of micromagnetism, a BP can be defined as a region inside a ferromagnet, where the magnetization collapses to zero [24]. On a shell that encloses the BP, any magnetization direction can be found [23]. In some cases, a $\mathrm{BP}$ is therefore similar to a magnetic hedgehog structure. In the micromagnetic simulations, the magnetization is calculated on discretization points, each of which has a well-defined magnetization direction. A BP in the simulations is therefore always located between the discretization points. It has been shown that BPs can be described reliably with micromagnetic simulations, provided that the grid size is fine enough $[25,26]$. Since the magnetization in the vicinity of a BP is maximally inhomogeneous, it is often difficult to determine the precise position of a BP in the simulations. In a similar way as the intersection of the $m_{x}=0$ and the $m_{y}=0$ isosurfaces has proven to be very helpful to locate the cores of (anti)vortices, the isosurfaces can greatly help to identify and locate a BP. In Figs. 3(f)- $3(\mathrm{j})$, the $m_{z}=0$ isosurface is displayed in addition to the $m_{x}=0$ and $m_{y}=0$ ribbons. A singularity occurs when these three isosurfaces intersect at one point: There, and only there, $|\boldsymbol{M}|$ is equal to zero inside the sample. In Fig. 3(i), the intersection of the cyan colored $m_{x}=0$, the green $m_{y}=0$, and the yellow $m_{z}=0$ ribbon marks the point where the magnetization locally vanishes. With this isosurface representation, we could clearly determine that the BP enters from the bottom surface [27] and runs through the layer within only 2-3 ps, which corresponds to a BP velocity between 300 and $500 \mathrm{~ms}^{-1}$. Compared to the typical speed of BPs driven by external fields, this value is unusually high. Because of their vanishing net magnetization, the mobility of BPs is typically very low $[24,26]$. Here the low BP mobility is compensated by the fact that the $\mathrm{BP}$ is driven by the strong exchange field, with typical values on the order of $100 \mathrm{~T}$, resulting in a high $\mathrm{BP}$ speed.

The spontaneous formation of a BP is an astonishing, previously unreported phenomenon in magnetism. A Bloch point is a region where the exchange energy density

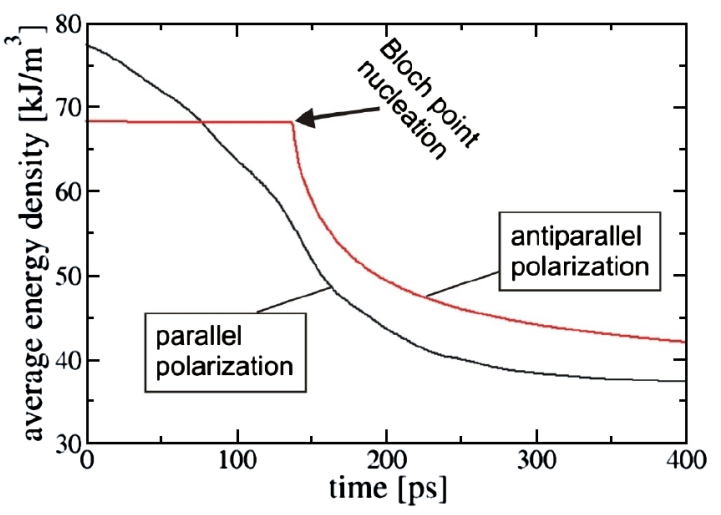

FIG. 4 (color online). Temporal evolution of the system's energy during the annihilation processes. In the case of antiparallel polarization, the formation of the BP initiates a rapid decay of the energy. In contrast to this, the energy decreases continuously during the annihilation process with parallel polarization. 
diverges. Nevertheless, the total energy of the sample remains finite [23]. Such a singularity is a highly energetic structure that usually requires a strong external driving force for its formation. While it is relatively well known that BPs can be injected by means of a strong external field $[25,26,28,29]$, most experts would probably agree that the activation-free formation of a $\mathrm{BP}$ is a counterintuitive result.

As can be seen in Fig. 4, the total energy of the sample hardly decreases until the BP is formed. Then, suddenly, the energy starts decaying exponentially. After the BP has left the sample on the top surface, the vortex-antivortex structure is completely dissolved and a large amount of exchange energy is released. This energy is immediately converted into spin waves, which can be seen as wave fronts being emitted from the pointlike region where the BP was formed. In a movie of this simulated annihilation process [30], the dynamics of the spin wave emission is particularly well visible. In this animated visualization, it can be seen that the emission of spin waves appears so suddenly and so strongly that it, in fact, resembles an explosion.

In conclusion, high-resolution micromagnetic simulations have been used to unveil the complicated magnetization dynamics of vortex-antivortex annihilation processes. When the polarization of the antivortex is opposite to the vortex polarization, the topology of the magnetization field is quite intriguing. In that case, the annihilation is mediated by a propagating BP. The details of the annihilation process occur on very small length scales of just a few nanometers. The rapid propagation and expulsion of a spontaneously formed BP has been identified as the source for spin waves formed in the annihilation process [10]. Besides the fundamental interest of this study, the simulations provide detailed insight into crucial aspects of the vortex core dynamics, which may be relevant for possible applications of these micromagnetic structures in magnetic data storage.

We thank Professor Manfred Fähnle for fruitful discussions.

[1] A. Hubert, J. Phys. (Paris), Colloq., Suppl. 49, C8-1859 (1988).

[2] A.S. Arrott and T. L. Templeton, Physica (Amsterdam) 233B, 259 (1997).

[3] A. Wachowiak, J. Wiebe, M. Bode, O. Pietzsch, M. Morgenstern, and R. Wiesendanger, Science 298, 577 (2002).

[4] J. Miltat and A. Thiaville, Science 290, 466 (2000).

[5] S. B. Choe, Y. Acremann, A. Scholl, A. Bauer, A. Doran, J. Stohr, and H. A. Padmore, Science 304, 420 (2004).

[6] J. P. Park, P. Eames, D. M. Engebretson, J. Berezovsky, and P. A. Crowell, Phys. Rev. B 67, 020403(R) (2003).

[7] A. Puzic, B. Van Waeyenberge, K. W. Chou, P. Fischer, H. Stoll, G. Schuetz, T. Tyliszcak, K. Rott, H. Brueckl, G. Reiss, I. Neudecker, T. Haug, M. Buess, and C.H. Back, J. Appl. Phys. 97, $10 \mathrm{E} 704$ (2005).
[8] K.-S. Lee, B.-W. Kang, Y.-S. Yu, and S.-K. Kim, Appl. Phys. Lett. 85, 1568 (2004).

[9] T. Okuno, K. Mibu, and T. Shinjo, J. Appl. Phys. 95, 3612 (2004).

[10] K.-S. Lee, S. Choi, and S.-K. Kim, Appl. Phys. Lett. 87, 192502 (2005).

[11] A. S. Arrott, J. Magn. Magn. Mater. 258-259, 25 (2003).

[12] R. Rahm, J. Stahl, and D. Weiss, Appl. Phys. Lett. 87, 182107 (2005).

[13] G.-W. Chern, H. Youk, and O. Tchernyshyov, J. Appl. Phys. 99, 08Q505 (2006).

[14] J. Raabe, R. Pulwey, R. Sattler, T. Schweinböck, J. Zweck, and D. Weiss, J. Appl. Phys. 88, 4437 (2000).

[15] E. E. Huber, Jr., D. O. Smith, and J. B. Goodenough, J. Appl. Phys. 29, 294 (1958).

[16] R. D. Gomez, T. Luu, A. Pak, K. Kirk, and J. Chapman, J. Appl. Phys. 85, 6163 (1999).

[17] R. Hertel, W. Wulfhekel, and J. Kirschner, Phys. Rev. Lett. 93, 257202 (2004).

[18] The visualization of the data is done with GMV, http:// www-xdiv.lanl.gov/XCM/GMVHome.html.

[19] Note that in Fig. 2 and elsewhere in this Letter, the choice of $t=0$ is to a certain extent arbitrary, since an unstable configuration is imposed artificially on the sample. The main purpose of the data concerning the time at which different snapshots are taken is to indicate the speed and the time scales relevant for the process.

[20] See EPAPS Document No. E-PRLTAO-97-068642 for a movie (movie_1) showing an isosurface representation of the dynamic conversion of a cross-tie structure into a vortex state in the case of parallel polarization of antivortex and vortices. For more information on EPAPS, see http://www.aip.org/pubservs/epaps.html.

[21] A. Hubert and R. Schäfer, Magnetic Domains-The Analysis of Magnetic Microstructures (Springer, Berlin, 1998).

[22] H. Kronmüller, Z. Phys. 168, 478 (1962).

[23] W. Döring, J. Appl. Phys. 39, 1006 (1968).

[24] E. Galkina, B. Ivanov, and V. Stephanovich, J. Magn. Magn. Mater. 118, 373 (1993).

[25] A. Thiaville, J. M. García, R. Dittrich, J. Miltat, and T. Schrefl, Phys. Rev. B 67, 094410 (2003).

[26] R. Hertel and J. Kirschner, J. Magn. Magn. Mater. 278, L291 (2004).

[27] The simulations indicate that, for this configuration, the BP always enters from the bottom surface. Further studies are required to determine whether the handedness of the vortex or its polarization are decisive for this.

[28] A.S. Arrott, B. Heinrich, and A. Aharoni, IEEE Trans. Magn. 15, 1228 (1979).

[29] J. Miltat and A. Thiaville, Science 298, 555 (2002).

[30] See EPAPS Document No. E-PRLTAO-97-068642 for a movie (movie_2) showing the dynamics of the annihilation process and the subsequent emission of spin waves in the case of antiparallel polarization of antivortex and vortices. Movie_3 shows a magnified view on the region of interest that shows that the vortex and the antivortex collapse to one point and thereby annihilate. For more information on EPAPS, see http://www.aip.org/pubservs/ epaps.html. 